Document downloaded from:

http://hdl.handle.net/10251/83347

This paper must be cited as:

Castellanos, W.; Guerri Cebollada, JC.; Arce Vila, P. (2016). Performance Evaluation of Scalable Video Streaming in Mobile Ad hoc Networks. IEEE Latin America Transactions. 14(1):122-129. http://hdl.handle.net/10251/83347.

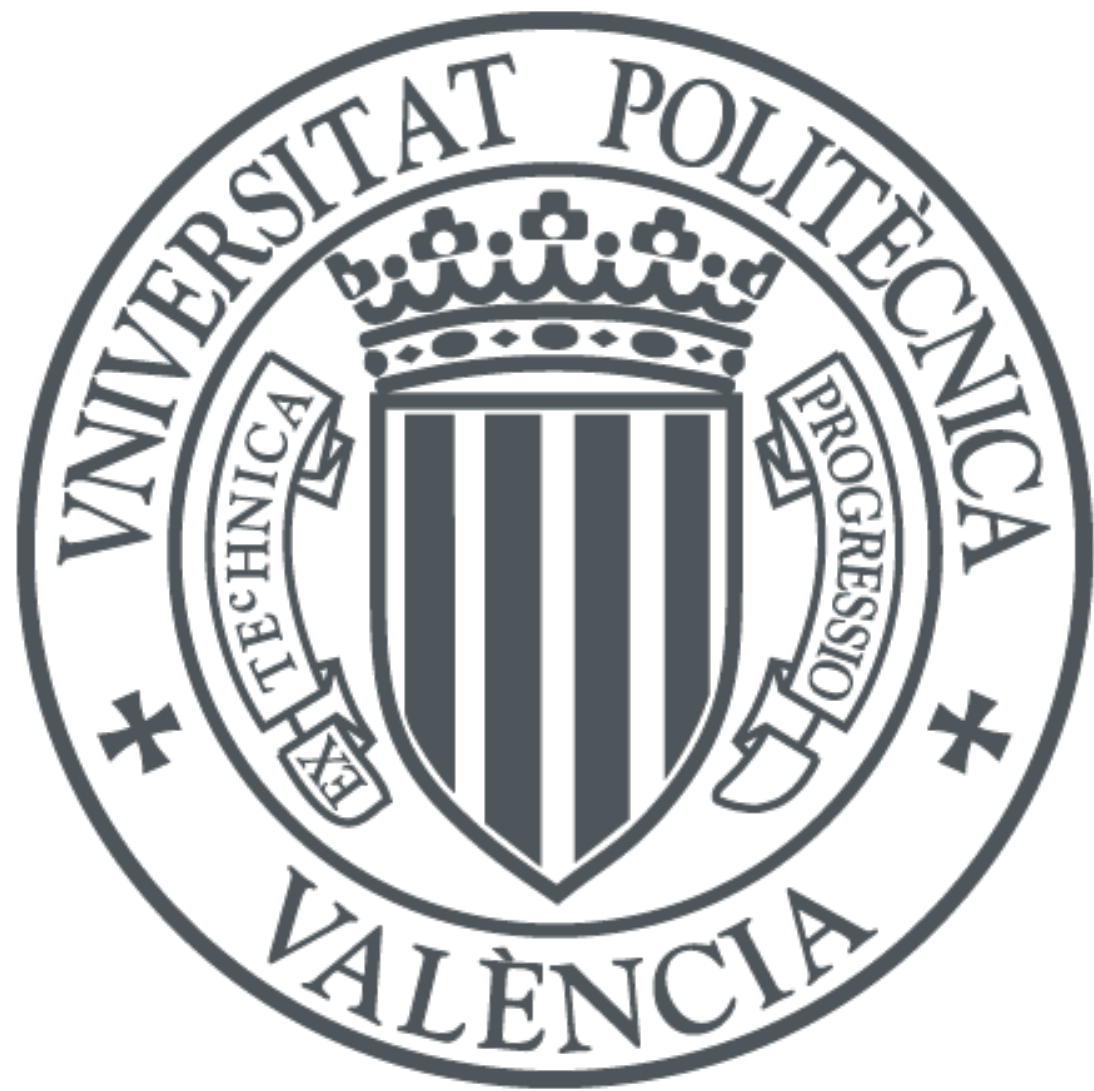

The final publication is available at

http://www.ewh.ieee.org/reg/9/etrans/eng/index.html

Copyright Institute of Electrical and Electronics Engineers (IEEE)

Additional Information

(c) 2016 IEEE. Personal use of this material is permitted. Permission from IEEE must be obtained for all other users, including reprinting/ republishing this material for advertising or promotional purposes, creating new collective works for resale or redistribution to servers or lists, or reuse of any copyrighted components of this work in other works. 


\title{
Performance Evaluation of Scalable Video Streaming in Mobile Ad hoc Networks
}

\author{
W. Castellanos, J.C. Guerri, P. Arce
}

\begin{abstract}
This paper presents a performance evaluation of the scalable video streaming over mobile ad hoc networks. In particular, we focus on the rate-adaptive method for streaming scalable video (H.264/SVC). For effective adaptation a new crosslayer routing protocol is introduced. This protocol provides an efficient algorithm for available bandwidth estimation. With this information, the video source adjusts its bit rate during the video transmission according to the network state. We also propose a free simulation framework that supports evaluation studies for scalable video streaming. The simulation experiments performed in this study involve the transmission of SVC streams with Medium Grain Scalability (MGS) as well as temporal scalability over different network scenarios. The results reveal that the rateadaptive strategy helps avoid or reduce the congestion in MANETs obtaining a better quality in the received videos.
\end{abstract}

Keywords - wireless communications, mobile ad hoc rnetworks, SVC video, scalable video streaming, rate-adaptive video streaming.

\section{INTRODUCCION}

$\mathrm{E}^{\mathrm{s}}$ L incremento de las capacidades de procesamiento de los dispositivos inalámbricos, así como las mejoras en la eficiencia energética de los mismos, ha provocado un incremento significativo en la transmisión de contenido multimedia proveniente de servicios de video por demanda, tal como YouTube o Vimeo. Debido a esto, el desarrollo de nuevas tecnologías de red para optimizar la transmisión de video sobre redes inalámbricas ha tenido un creciente interés. Entre los diferentes tipos de redes inalámbricas, se espera que las redes móviles ad hoc o MANETs (Mobile Ad hoc NETworks) sean ampliamente utilizadas en el futuro cercano, en diversos escenarios y bajo diferentes paradigmas [1]. Las MANETs están conformadas por nodos móviles que establecen comunicaciones entre sí, sin contar con una estación base o infraestructura cableada. Por lo tanto, cada nodo se apoya en los demás para conseguir comunicarse con otro nodo fuera de su rango de alcance, creando la llamada comunicación multi-salto [2]. Sin embargo, la trasmisión de video por este tipo de redes no es una tarea fácil, principalmente debido a la movilidad de los nodos y al hecho de que el canal inalámbrico es compartido entre nodos vecinos. Si adicionalmente se usa la técnica tradicional de transmisión de video, donde este se transmite a una tasa de

W. Castellanos, Instituto de Telecomunicaciones y Aplicaciones Multimedia (iTEAM), Universitat Politècnica de València, Valencia, España, wilcashe@upv.es

J.C. Guerri, Instituto de Telecomunicaciones y Aplicaciones Multimedia (iTEAM), Universitat Politècnica de València, Valencia, España, jcguerri@dcom.upv.es

P. Arce, Instituto de Telecomunicaciones y Aplicaciones Multimedia (iTEAM), Universitat Politècnica de València, Valencia, España, paarvi@iteam.upv.es bits constante, la calidad del video recibido puede sufrir una degradación significativa. Por lo tanto, se deben aplicar nuevos esquemas de transmisión más flexibles y robustos. Una posible solución es la utilización de la codificación escalable de video (H.264/SVC, Scalable Video Coding) [3]. Un video codificado de acuerdo a H.264/SVC está compuesto por varias capas (una capa base y varias capas de "mejora") que proveen diferentes niveles de calidad. Con este esquema por capas se obtiene: $i$ ) flujos codificados con mayor robustez en entornos con altas pérdidas de paquetes y ii) la posibilidad de adaptar el flujo de video a las condiciones de la red por medio de la eliminación o adición de capas.

En este artículo se desarrolla una evaluación de dos esquemas de transmisión de video escalable sobre las redes MANETs. Un esquema consiste en transmitir el video manteniendo una tasa de transmisión fija pero enviando la información de todas las capas. El otro esquema incorpora un modelo adaptativo, en el cual la fuente de tráfico elimina capas del flujo SVC para adaptarse al ancho de banda disponible. También se proponen dos herramientas complementarias para el estudio de tráfico SVC sobre redes de datos. Por un lado, se propone un nuevo protocolo de encaminamiento con comunicación inter-capas, para estimar e informar a la fuente los recursos disponibles en la red (en términos de ancho de banda disponible). Por otra parte, se expone una nueva plataforma de simulación llamada SVCEval-RA (SVC Evaluation platform for Rate-Adaptive Video). Esta herramienta software permite simular la transmisión de video escalable sobre redes inalámbricas.

Los resultados de las simulaciones permiten demostrar que la estrategia adaptativa evita o ayuda a reducir la congestión de la red y la pérdida de paquetes, obteniendo una mejor calidad en los videos recibidos.

La organización del artículo es la siguiente: en la Sección II se describen algunos trabajos relacionados. En la Sección III se presenta un resumen de los aspectos más importantes de la transmisión adaptativa de video escalable. La Sección IV describe las principales características de la plataforma de simulación desarrollada. La Sección V presenta el protocolo de encaminamiento propuesto. En las Secciones VI y VII se describe el entorno de evaluación y los resultados, respectivamente. Finalmente, se plantean algunas conclusiones y algunas líneas de trabajo futuro en la Sección VIII.

\section{TRABAJOS RELACIONADOS}

Diferentes soluciones basadas en SVC han sido planteadas recientemente para la transmisión de video. Por ejemplo, los trabajos de Famaey et al. [4] y Kalva et al. [5] muestran las ventajas de usar SVC en la transmisión adaptativa de video 
sobre HTTP frente a la codificación de capa única (AVC, Advanced Video Coding). Xiang et al. [6] desarrollan un algoritmo para la adaptación de flujos SVC durante la transmisión de video sobre redes inalámbricas usando DASH (Dynamic Adaptive Streaming over HTTP) [7].

Pero no solamente sobre HTTP puede darse la transmisión de videos SVC. La flexibilidad y robustez del esquema por capas de la codificación escalable, ha motivado el desarrollo de diversas propuestas para integrar SVC con otras tecnologías de red. Por ejemplo, Sanna e Izquierdo [8] plantean la transmisión de video SVC en redes peer-to-peer junto con técnicas de codificación de red, Aladbulkarim et al. [9] implementan una solución para la transmisión de video SVC sobre redes ZigBee, Yaacoub et al. [10] proponen un esquema para mejorar la transmisión de video escalable en redes vehiculares y en [11] se estudia el uso de SVC en redes móviles LTE.

En relación con las redes móviles ad hoc, Halloush et al. [12] y Rhaiem et al. [13] evalúan el funcionamiento de los protocolos de encaminamiento convencionales de las MANETs, durante la transmisión de flujos SVC. Los resultados de estos estudios, demuestran la necesidad de implementar nuevos mecanismos de red con el fin de proporcionar ciertos niveles de calidad de servicio (QoS). Una solución enfocada a proveer QoS en MANETs para la transmisión de video SVC es la presentada por Lal et al. [14], en la cual se implementa un nuevo protocolo de encaminamiento que incluye un control de admisión basado en la estimación del ancho de banda disponible. También está el trabajo presentado por Radu et al. [15], que introduce el monitoreo de la calidad subjetiva y a la vez un método de protección de errores durante la transmisión del video SVC. Para contrarrestar las altas pérdidas de paquetes que pueden presentarse en las MANETs, Lai y Liou [16] proponen un esquema basado en la asignación de prioridades a los paquetes de video. Sin embargo, las soluciones para MANETs propuestas hasta el momento, no han evaluado con profundidad la opción de adaptar la tasa de transmisión, mediante la eliminación o adición de capas SVC, de acuerdo a la estimación de los recursos disponibles en la red. Además, este esquema de adaptación no podría ser evaluado con las herramientas tradicionales, ya que estas solo permiten simular la distribución de tráfico multimedia a una tasa constante. Esto desaprovecha el esquema por capas de SVC. Por tanto, se hace necesario el desarrollo nuevas plataformas de simulación, tal como la herramienta propuesta en este artículo. Por otro lado, las soluciones para MANETs estudiadas, solo evalúan la escalabilidad temporal y espacial que ofrece SVC, pero descartan la escalabilidad de calidad. En este artículo, no solo se utiliza la combinación de la escalabilidad temporal y de calidad, sino que además se usa el esquema MGS (Medium Grain Scalability), que es el esquema de escalabilidad de calidad que mejores prestaciones ofrece, tal como lo demuestran numerosos estudios, por ejemplo [17] y [18].

\section{TRANSMisión AdAPTATIVA dE VideO EscALABLE}

La codificación escalable H.264/SVC [3] permite la codificación de un video mediante la creación de "capas" que conforman un único flujo de datos. La más básica representación del video está contenida en la "capa base" la cual consiste en la representación de más baja calidad. También se generan capas de "mejora" que incrementan la calidad cuando son añadidas a la capa base. Este esquema por capas de SVC, provee una alta robustez durante la transmisión de video sobre redes con constantes fluctuaciones del ancho de banda, ya que permite al transmisor adaptar su tasa de envío de datos, añadiendo o eliminando capas del video SVC, de acuerdo a la estimación del ancho de banda disponible (ver Fig. 1). Por lo tanto, para poder adaptar dinámicamente el flujo de video, es necesario contar con un mecanismo que haga una estimación del ancho de banda disponible y lo informe a la fuente del video. En este artículo se propone el uso del protocolo AQA-AODV, el cual es una solución intercapas (cross-layer) que permite: estimar el ancho de banda disponible, intercambiar información entra las capas (de red, transporte y aplicación) y establecer rutas dentro de la red MANET. También permite hacer una realimentación hacia la capa de aplicación para informar de los recursos disponibles en la red.

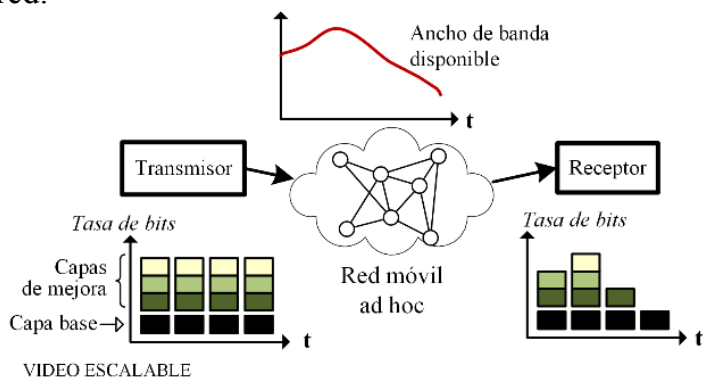

Figura 1. Transmisión adaptativa de video escalable

\section{A. Codificación escalable de video (SVC)}

La codificación SVC hace parte del estándar H.264/MPEG4. Un video que ha sido codificado siguiendo el esquema de H.264/SVC tiene una estructura por capas, las cuales corresponden a diferentes niveles de calidad o a diferentes representaciones espaciales o temporales. El principal aporte de la codificación SVC es el poder contar con tres diferentes tipos de escalabilidad (ver Fig. 2): espacial, temporal y de calidad (también llamada escalabilidad SNR- signal-to-noiseratio).

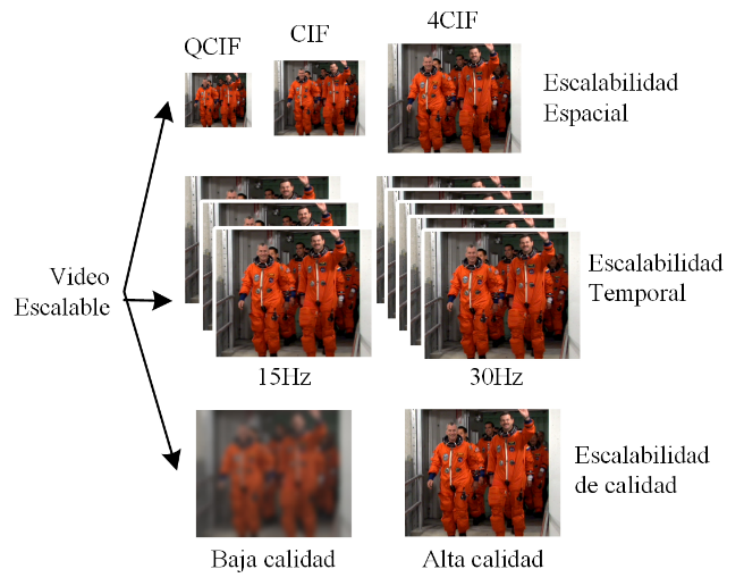

Figura 2. Diferentes tipos de escalabilidad en H.264/SVC

El término escalabilidad, en el contexto de SVC, significa que la información del video puede ser recuperada 
decodificando solamente una parte del video codificado. Por ejemplo, es posible decodificar el video con una menor resolución que el original, decodificando solo la capa espacial más baja y descartando las otras capas espaciales.

Cuando se usa la escalabilidad temporal, las capas mejoran la tasa de cuadros (frame rate). Con la escalabilidad espacial la capa base se codifica a una resolución espacial baja (tamaño del fotograma) y las capas de mejora ofrecen un incremento progresivo de la resolución (ver Fig. 2). Finalmente, la escalabilidad en calidad (o SNR) se refiere a escalar en términos del nivel de compresión aplicado al video original. Con la escalabilidad por calidad, la capa base contiene una versión muy comprimida de cada fotograma y las capas de mejora incorporan más información para incrementar el valor de la relación señal a ruido (SNR Signal-Noise-Ratio).

El estándar H.264/SVC permite la escalabilidad combinada, es decir, un video escalable puede usar una combinación de los tres tipos de escalabilidad. Por ejemplo, la Fig. 3 muestra la estructura de un video SVC con tres niveles temporales $\left\{\mathrm{T}_{0}, \mathrm{~T}_{1} \mathrm{y} \mathrm{T}_{2}\right\}$ y dos niveles de calidad $\left\{\mathrm{Q}_{0} \mathrm{y}_{1}\right\}$. Por lo tanto se generan 8 capas de acuerdo a la combinación de estos niveles, tal y como se describe en la Fig. 3. La capa base está conformada por la capa de más baja resolución temporal $\mathrm{T}_{0}$ y del nivel de calidad más bajo (es decir, de los cuadros 0 y 4). Un ejemplo de una capa de mejora puede ser la capa conformada por el nivel temporal $\mathrm{T}_{2} \mathrm{y}$ el nivel de calidad $\mathrm{Q}_{1}$.

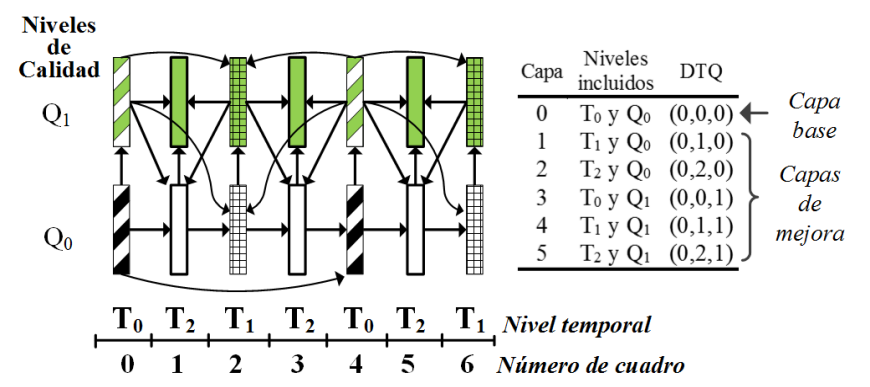

Figura 3. Ejemplo de una estructura de codificación SVC con escalabilidad temporal y de calidad.

En H.264/SVC el codificador está dividido en dos subsistemas: el subsistema de codificación de video (VCL, Video Coding Layer) y el subsistema de adecuación a la red (NAL, Network Abstraction Layer). Básicamente, el subsistema VCL se encarga de la codificación del video y el subsistema NAL convierte la representación que hace VCL del video en el formato correcto de acuerdo a la capa de transporte. La información del video codificado se organiza en unidades de almacenamiento llamadas NALUs (Network Abstraction Layer Units). Las NALUs contienen los datos codificados de los fotogramas, parcial o completamente. Cada NALU tiene tres identificadores: dependency identifier (DID), temporal identifier (TID) y quality identifier (QID). Estos identificadores representan el nivel de escalabilidad espacial, temporal y de calidad, respectivamente. Los valores DID, TID y QID también son conocidos como parámetros (D,T,Q). Por ejemplo, una NALU que transporta información de la capa base, debe estar identificada como $(0,0,0)$ y la capa de mejora conformada por los niveles $\mathrm{T}_{2}$ y $\mathrm{Q}_{1}$ se identificaría como
$(0,2,1)$. La inspección de los parámetros DTQ permite identificar a que capa pertenece una determinada NALU. Este hecho es particularmente importante, ya que de esta manera una NALU puede ser identificada y removida del flujo de video SVC para reducir la tasa de bits. A pesar de la eliminación de algunas NALUs, las NALUs restantes podrían servir para conformar un video correctamente decodificable pero con una menor calidad. Sin embargo, las dependencias de las capas también se deben tener en cuenta. Las capas en SVC pueden ser decodificadas independientemente pero hay una lógica dependencia entre ellas. Esta interdependencia debe ser considerada para lograr una correcta decodificación del video. En el ejemplo que se describe en la Fig. 3, las flechas representan las dependencias entre los cuadros (frames) que componen un video con escalabilidad combinada (temporal y de calidad). Por ejemplo, el cuadro 1 de la capa $\mathrm{T}_{2} \mathrm{Q}_{0}$ depende de la capa $\mathrm{T}_{0} \mathrm{Q}_{1}$ y también de la capa $\mathrm{T}_{1} \mathrm{Q}_{1}$. Debido a estas dependencias, descartar una capa de calidad de un cuadro de referencia (e.g. el cuadro 2) afecta la calidad de los cuadros dependientes (e.g. los cuadros 1 y 3 ).

\section{a) Escalabilidad de calidad $M G S$}

El estándar H.264/SVC provee tres tipos de escalabilidad por calidad: escalabilidad por granularidad gruesa (CGS, Coarse Grain Scalability), escalabilidad por granularidad media (MGS, Medium Grain Scalability) y escalabilidad por granularidad fina (FGS, Fine Grain Scalability) [18]. En este artículo se utilizará la escalabilidad MGS ya que este tipo de escalabilidad incrementa la eficiencia de codificación y permite que se pueda cambiar de capa en cualquier momento de la decodificación. En la Fig. 4 se describe la ventaja de usar MGS frente a otros esquemas de escalabilidad. Con el esquema CGS cada capa de mejora es estimada por separado. Este esquema tiene varias desventajas. Por ejemplo, si una parte de la capa de mejora se pierde entonces se obtiene un error en la estimación de la secuencia posterior (ver Fig. 4a). Con el esquema de FGS cada capa de mejora se predice a partir de la capa base (Fig. 4b). Con ello se evita que si se pierden los paquetes correspondientes a la capa de mejora durante unos instantes de tiempo, se pueda continuar decodificando la capa de mejora a partir de la capa base. Sin embargo, tenemos una eficiencia de codificación muy baja. La mejor solución es el esquema MGS Fig. 4c ya que hay más flexibilidad al momento de elegir cuál de las capas de calidad se puede usar para la predicción. Además, se introduce el concepto de los fotogramas de referencia, que sirven para hacer actualizaciones periódicas en la capa base. En la referencia [18] se puede consultar información más detallada sobre la técnica de codificación de video escalable y los diferentes esquemas de escalabilidad.

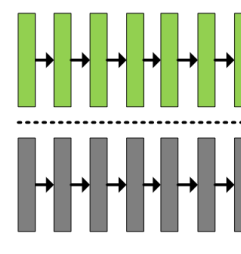

(a) CGS

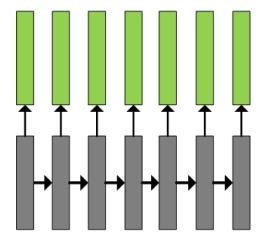

(b) FGS

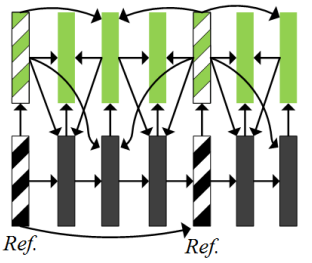

(c) MGS
Figura 4. Métodos de escalabilidad de calidad 


\section{DESCRIPCIÓN DE LA PLATAFORMA DE SIMULACIÓN SVCEVAL-RA}

A continuación se hace una descripción la plataforma de simulación propuesta, llamada SVCEval-RA (SVC Evaluation platform for Rate-Adaptive Video). Esta plataforma la hemos desarrollado con el objetivo de evaluar la transmisión de video adaptativo codificado en SVC sobre redes de datos. Recientemente, han sido desarrolladas algunas herramientas software para simular la transmisión y evaluación de video escalable. Por ejemplo, está la plataforma SVEF (Scalable Video-streaming Evaluation Framework) [19] la cual es un conjunto de pequeñas aplicaciones que permiten la evaluación de flujos SVC en tiempo real. Sin embargo, SVEF tiene una limitada aplicación ya que no permite realizar experimentos sobre nuevas tecnologías de red o usando diversos protocolos. Tampoco permite usar la escalabilidad espacial y solo soporta videos SVC con una sola capa de dependencia. Otras plataformas para la transmisión de videos escalables son EvalSVC (Evaluation of $S V C$ ) y myEvalSVC, las cuales fueron desarrolladas por Le et al. [20] y Ke [21], respectivamente. Estas plataformas de simulación están integradas al simulador de redes NS-2 y por tanto, permiten simular la transmisión de video SVC usando las diferentes tecnologías de red incluidas en dicho simulador. Sin embargo, estas plataformas no contienen los algoritmos necesarios para que la fuente de video escalable adapte su tasa de bits, descartando o añadiendo las capas que no pueden ser soportadas por la red. A diferencia de las plataformas existentes, SVCEval-RA contiene los mecanismos necesarios para que los flujos de video escalable puedan ajustar su tasa de bits de acuerdo al ancho de banda disponible en la red. Además, SVCEval-RA ha sido integrada en el simulador de redes NS-2, de tal manera que pueda ser usada junto con nuestro protocolo AQA-AODV para evaluar las ventajas obtenidas en la transmisión de vídeo sobre redes móviles ad hoc. La Fig. 5 muestra un diagrama de flujo de la herramienta de simulación SVCEval-RA. Principalmente consiste en tres principales fases: codificación, simulación y decodificación. En la fase de codificación el video original en formato YUV es codificado de acuerdo al estándar H.264/SVC. La codificación se realiza por medio del software JSVM (Joint Scalable Video Model) [22]. Durante esta fase se configuran todas las características que tendrá el video codificado, tal como el tipo de escalabilidad y el número de capas. Posteriormente, por medio de la herramienta BitStreamExtractor, incluida en JSVM, se genera un fichero de texto (traza de NALUs) con información acerca de los paquetes que conforman cada NALU. Algunos de los parámetros que almacena son: el tamaño del paquete, los valores DTQ y el tipo de paquete. Datos que son necesarios para la posterior decodificación del video. Antes de ejecutar la fase de simulación, también es necesario generar dos trazas adicionales. Estas trazas se generan a través del software SVCEval-RA. Por un lado se genera el fichero traza de bitrates, la cual contiene información acerca de la tasa de transmisión requerida para cada capa del video codificado en SVC. Específicamente, los datos que contiene son: identificador de capa, tasa de cuadros (frames por segundo), tasa de transmisión (Kbps), los parámetros DTQ y el PSNR (dB). Por otra parte, se genera la traza de tráfico NS-2, la cual es una adaptación de la traza de NALUs al formato aceptado por NS-2.

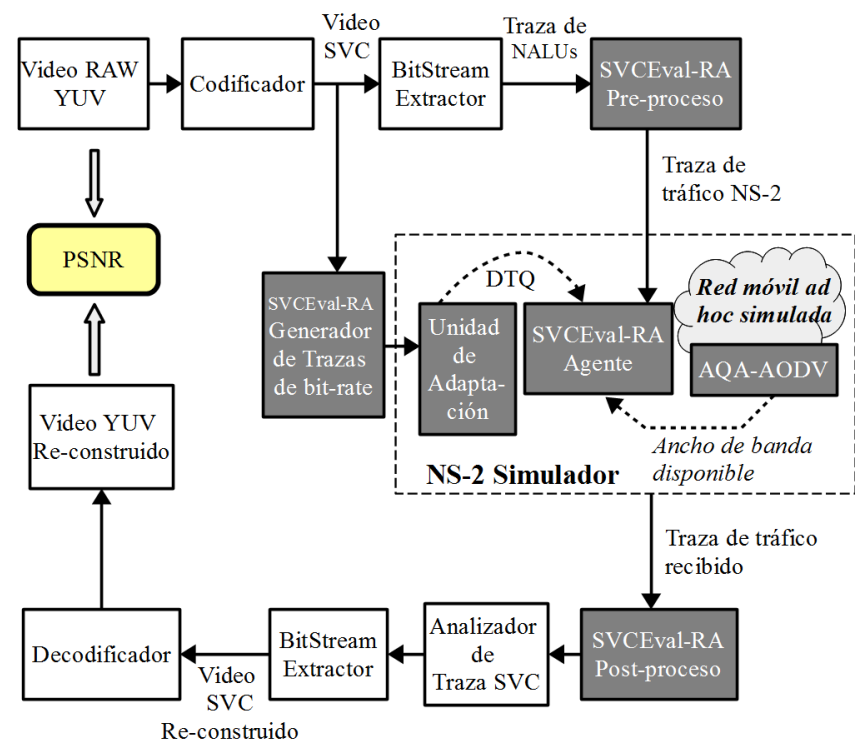

Figura 5 Representación esquemática de la plataforma SVCEval-RA.

Durante la fase de simulación, la información contenida en el fichero traza de tráfico NS-2 se utiliza para generar los paquetes y transmitirlos sobre la red móvil ad hoc simulada. Como resultado de la simulación, se obtiene un fichero (traza de tráfico recibido), similar al fichero de entrada (es decir, un registro por cada paquete), pero adicionalmente se indica la latencia experimentada por cada paquete durante su transmisión. Si un paquete se pierde durante el envío, el correspondiente registro no aparecerá en el fichero de salida. Durante la transmisión de los paquetes de video por la red móvil ad hoc, nuestro protocolo AQA-AODV provee la estimación del ancho de banda disponible, la cual es enviada a la unidad de adaptación. En dicho módulo, se determinan los parámetros DTQ de la capa más alta que puede transmitirse sin superar la limitación del ancho de banda. Por lo tanto, la capa seleccionada es aquella con un bit-rate menor o igual que la tasa de transmisión soportada por la red. A partir de los parámetros DTQ, el agente de software SVCEval-RA integrado en el simulador, filtra los paquetes correspondientes a dicha capa (y sus capas inferiores), para que solo estos sean transmitidos por la red móvil ad hoc.

Después de la simulación el fichero traza de tráfico recibido es procesado por SVCEval-RA para obtener la traza de NALUs recibidas. Esta última traza necesita ser filtrada: i) para eliminar las NALUs que han superado el límite de retardo, ii) reordenar las NALUs de acuerdo al orden de envío y iii) eliminar las NALUs que no tienen satisfechas sus dependencias para la decodificación.

Después de filtrar la traza de NALUs recibidas, y con ayuda de la herramienta BitStreamExtractor, se reconstruye el video codificado en SVC, el cual posteriormente de decodifica. El video resultante estará nuevamente en formato YUV y por lo tanto puede ser comparado el video YUV 
original con el fin de medir la calidad del video recibido (cálculo del PSNR). Adicionalmente, otras métricas pueden ser calculadas a partir de la traza de tráfico NS-2, tal como la tasa de paquetes perdidos y el retardo.

\section{AQA-AODV: PROTOCOLO DE ENCAMINAMIENTO CON OoS PARA REDES MOVILES AD HOC}

Además de desarrollar la plataforma de simulación SVCEval-RA para evaluar la trasmisión de video SVC sobre redes MANETs, también hemos implementado un protocolo de encaminamiento que aporta mecanismos que permiten ofrecer ciertos niveles de calidad de servicio. En esta sección se describe el protocolo desarrollado, el cual ha sido denominado AQA-AODV (Adaptive QoS-Aware Ad-hoc Ondemand Distance Vector). Este protocolo ha sido desarrollado como una modificación del protocolo AODV. Las principales modificaciones afectan el proceso de búsqueda de rutas y la estrategia de mantenimiento de rutas de AODV.

Para realizar dichas modificaciones, fueron introducidos nuevos campos en los paquetes usados en AODV. Por ejemplo, al paquete RREQ (paquete de petición de ruta) fue incorporado un nuevo campo para informar el ancho de banda requerido y otro para el identificador de sesión (SessionID), los cuales, son utilizados para identificar cada flujo que se cursa por la red. También al paquete RREP (paquete de respuesta de petición) y el RERR (paquete de error de ruta) fueron modificados con nuevos campos. Los nuevos paquetes en AQA-AODV han sido llamados QRREQ, QRREP y QRRER para enfatizar la nueva función de transportar parámetros de QoS.

La principal diferencia de AQA-AODV con otras soluciones basadas en AODV es el esquema de realimentación adaptativa por el cual el nodo fuente puede adaptar su tasa de trasmisión de acuerdo al estado de la ruta. Por esta razón, los nodos a lo largo de la ruta deben incorporar algoritmos que les permita estimar la disponibilidad de recursos (en términos de ancho de banda).

Los principales aportes de AQA-AODV son:

i) Un algoritmo para la estimación del ancho de banda disponible en la red mediante la modificación del formato del mensaje HELLO y la creación de un nuevo mensaje llamado HELLOACK..

ii) Un agente "cross-layer" que permite intercambiar información entre la capa de aplicación y la capa de red. Mediante este agente se informa a la capa de red, el ancho de banda solicitado por la capa de aplicación y de igual manera el protocolo de encaminamiento informa a la fuente de tráfico, la tasa a la cual puede transmitir.

iii) Un nuevo procedimiento de descubrimiento de rutas (procesamiento del mensaje QREEQ) y del procedimiento de respuesta (procesamiento del mensaje QRREP) que tenga en cuenta los nuevos parámetros (ancho de banda disponible, identificador de sesión y máxima tasa de transmisión) en el establecimiento de rutas. iv) Un nuevo esquema de mantenimiento y recuperación de rutas, el cual incorpora una tabla caché con todas las sesiones activas.

v) Modificación de la caché de vecinos y de la tabla de encaminamiento, para incorporar el parámetro de calidad de servicio y el identificador de sesión.

La Fig. 6 muestra un diagrama con los principales módulos funcionales de AQA-AODV. Los tres principales elementos de AQA-AODV son: el módulo de estimación del ancho de banda, el módulo de establecimiento de rutas y el módulo de recuperación de rutas. El primer módulo realiza la estimación del ancho de banda disponible y provee realimentación de dicho valor a la capa de aplicación. Los paquetes HELLO son usados para la realizar dicha estimación. Estos paquetes son usados originalmente en AODV en el descubrimiento de nodos vecinos. Con la información sobre el ancho de banda disponible, la fuente de tráfico puede adaptar su tasa de transmisión, tal y como se propone en este artículo. Por otro lado, el módulo de establecimiento de rutas recibe la petición de rutas proveniente de la fuente de tráfico, en la cual se indica la tasa de transmisión requerida, y ejecuta el proceso de búsqueda de rutas. El tercer módulo es el módulo de recuperación de rutas, el cual se encarga de detectar fallos de conectividad y re-establecer rutas hacia el destino, teniendo en cuenta las condiciones de QoS previamente establecidas. Para obtener información más detallada sobre el funcionamiento de los diferentes procesos de AQA-AODV pueden ser consultada la referencia [23].

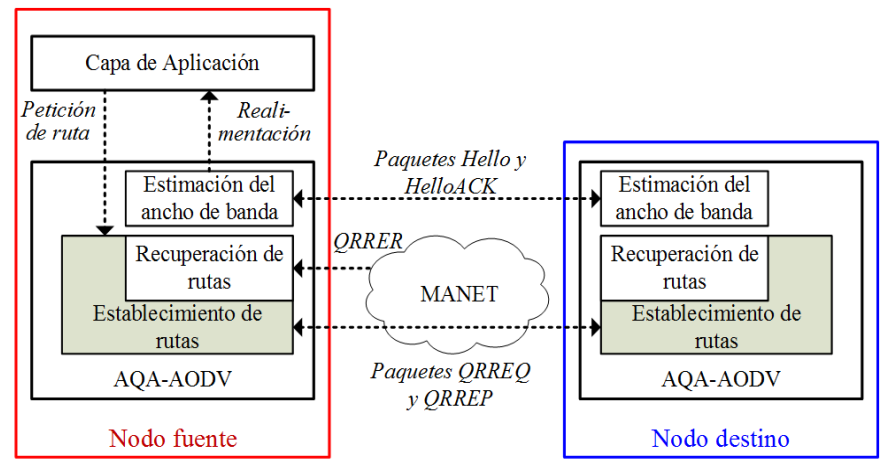

Figura 6. Diagrama funcional de AQA-AODV

\section{EVALUACIÓN}

En esta sección se describen los parámetros utilizados durante las simulaciones. El principal objetivo de las simulaciones es evaluar dos estrategias de transmisión de video escalable sobre MANETs: SVC Adaptativo y SVC Noadaptativo. La estrategia adaptativa utiliza el protocolo AQAAODV como protocolo de encaminamiento y la estrategia no adaptativa el protocolo AODV. También, se pretende mostrar cómo la integración de nuestras herramientas (AQA-AODV y SVCEval-RA) contribuye al estudio de la transmisión de videos adaptativos sobre redes móviles ad hoc.

Las simulaciones se desarrollaron sobre dos escenarios. El primer escenario es una red inalámbrica ad hoc con nodos estáticos y el otro escenario consiste en una red móvil ad hoc. 


\section{A. Entorno de simulación}

Para realizar la evaluación del tráfico SVC sobre MANETs, fue utilizado el simulador de redes NS-2. En la Tabla I se describen los parámetros configurados en el simulador.

TABLA I. PARÁMETROS DE SIMULACIÓN.

\begin{tabular}{ll}
\hline \hline \multicolumn{1}{c}{ CARACTERÍSTICA } & \multicolumn{1}{c}{ VALOR } \\
\hline Protocolo MAC & 802.11 \\
Modelo de propagación & Two Ray Ground \\
Tipo de cola & Drop Tail \\
Tamaño del paquete UDP & 1000 bytes \\
Rango de Transmisión & $250 \mathrm{~m}$ \\
Rango de Interferencia & $550 \mathrm{~m}$
\end{tabular}

La secuencia de video utilizada como flujo de tráfico se generó concatenando tres veces el video "CREW". Este video hace parte de la librería de videos de pruebas de Xiph.org Foundation [24] y es ampliamente utilizado en pruebas de simulación. El video concatenado se codificó de acuerdo al estándar H.264/SVC con dos tipos de escalabilidad: temporal y de calidad (MGS). El códec SVC utilizado fue el JSVM códec [22]. Otros parámetros relacionados con la codificación del video están resumidos en la Fig. 7.

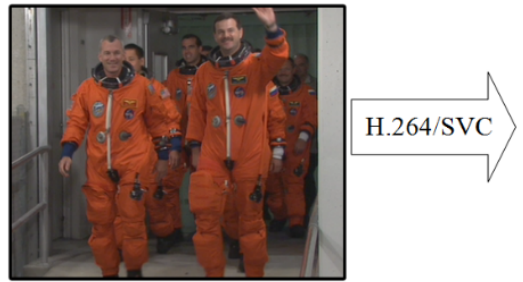

Formato: YUV

Resolución: CIF (352x288)

30 fotogramas/segundo

300 fotogramas

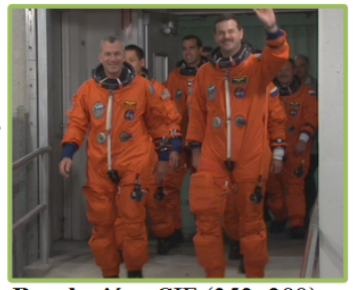

Resolución: CIF (352x288)

Escalabilidad: Temporal y

de calidad (MGS)

300 fotogramas
Figura 7. Características del video

El flujo SVC generado contiene 5 niveles temporales, identificados como $\mathrm{T}_{0}, \mathrm{~T}_{1}, \mathrm{~T}_{2} \mathrm{~T}_{3}$ y $\mathrm{T}_{4}$. Cada nivel temporal tiene 4 niveles de calidad $\left(\mathrm{Q}_{0} \mathrm{Q}_{1} \mathrm{Q}_{2}\right.$ y $\left.\mathrm{Q}_{3}\right)$. En total se obtuvieron 20 capas $\left(\mathrm{L}_{0}-\mathrm{L}_{19}\right)$ a partir de la combinación de los niveles $\mathrm{T}_{\mathrm{i}}$ y $\mathrm{Q}_{\mathrm{j}}$. Dependiendo del número de capas transmitidas, la tasa de bits varía desde $94.4 \mathrm{Kbps}$ (transmitiendo solo la capa 0) a 1 Mbps (enviando todas las 20 capas). En la Tabla II se muestra la tasa de bits que está asociada a cada capa. Estos valores son acumulados, es decir, dichas tasas de bits no solamente tienen en cuenta la tasa necesaria para transmitir los datos de una capa específica, sino que además incluye la tasa de bits necesaria para transmitir las capas de las cuales depende. Por ejemplo, para transmitir la capa 3 la tasa de bits total es de $273.3 \mathrm{Kbps}$ (ver Tabla II) esta tasa incluye la capa 3 y las capas inferiores de las cuales depende (capas 0, 1, y 2).

En los dos escenarios simulados se calculó el porcentaje de paquetes perdidos, el PSNR de la luminancia y las tasa de fotogramas decodificados. Esta última métrica corresponde al número de fotogramas satisfactoriamente decodificados divido entre el número total de fotogramas.
TABLA II. DESCRIPCIÓN DE LAS CAPAS DEL VIDEO CODIFICADO EN SVC

\begin{tabular}{|c|c|c|c|}
\hline $\begin{array}{l}\text { CAPA } \\
\text { ID }\end{array}$ & Ti Qi & $\begin{array}{c}\text { TASA DE } \\
\text { FRAMES } \\
(\mathrm{Hz})\end{array}$ & $\begin{array}{l}\text { TASA DE BITS } \\
\text { ACUMULADA }\end{array}$ \\
\hline 0 & T0 Q0 & 1.875 & 94.4 \\
\hline 1 & T1 Q0 & 3.75 & 139.9 \\
\hline 2 & T2 Q0 & 7.5 & 197.4 \\
\hline 3 & T3 Q0 & 15 & 273.3 \\
\hline 4 & T4 Q0 & 30 & 335.4 \\
\hline 5 & T0 Q1 & 1.875 & 157.0 \\
\hline 6 & T0 Q2 & 1.875 & 197.4 \\
\hline 7 & T0 Q3 & 1.875 & 245.1 \\
\hline 8 & T1 Q1 & 3.75 & 254.9 \\
\hline 9 & T1 Q2 & 3.75 & 314.1 \\
\hline 10 & T1 Q3 & 3.75 & 376.7 \\
\hline 11 & $\mathrm{~T} 2 \mathrm{Q} 1$ & 7.5 & 401.5 \\
\hline 12 & T2 Q2 & 7.5 & 487.0 \\
\hline 13 & T2 Q3 & 7.5 & 569.1 \\
\hline 14 & T3 Q1 & 15 & 593.0 \\
\hline 15 & T3 Q2 & 15 & 707.1 \\
\hline 16 & T3 Q3 & 15 & 808.7 \\
\hline 17 & T4 Q1 & 30 & 809.3 \\
\hline 18 & T4 Q2 & 30 & 948.7 \\
\hline 19 & T4 Q3 & 30 & 1066.8 \\
\hline
\end{tabular}

\section{Resultados}

\section{A. Escenario 1: Cadena de nodos estáticos de longitud variable}

El escenario 1 es una red inalámbrica cuyos nodos permanecen estáticos y situados según una topología lineal (ver Fig. 8). El nodo 1 es la fuente de video y el último nodo de la cadena es el nodo receptor. En este escenario el número de nodos de la cadena va aumentando desde 2 hasta 15 nodos. El objetivo es evaluar la adaptación de la tasa de bits de la fuente de tráfico de acuerdo al número de nodos.

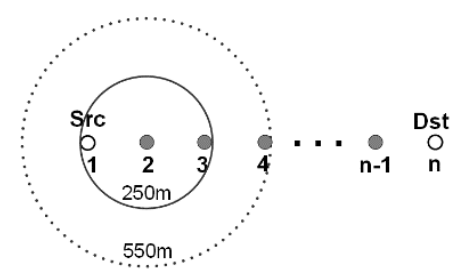

Figura 8. Escenario 1: cadena de nodos estáticos.

En la Fig. 9 se muestran los resultados de la simulación, tal como se describe a continuación: la variación del ancho de banda disponible estimado por AQA-AODV en función del número de nodos que conforman la red se describe en la Fig.9a; la Fig. 9b muestra el porcentaje de paquetes perdidos, la Fig. 9c describe la variación la tasa de fotogramas decodificados y la Fig. 9d la calidad del video recibido, en términos del PSNR de la luminancia (Y-PSNR). Cada figura muestra los resultados obtenidos, tanto para la transmisión de video SVC adaptativo, como para la transmisión NoAdaptativa. Estas curvas varían en función del número de nodos que conforman la ruta entre el nodo fuente y el receptor. 


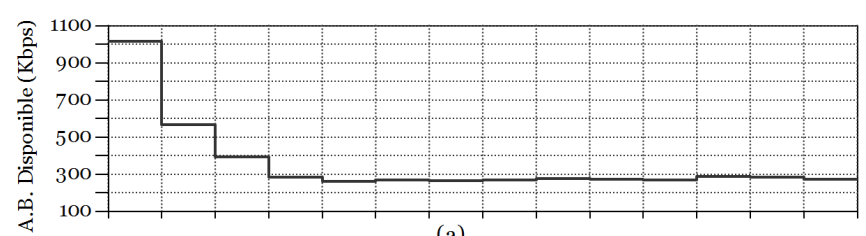

(a)

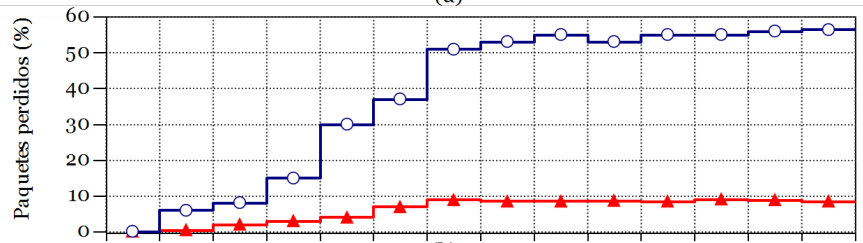

(b)
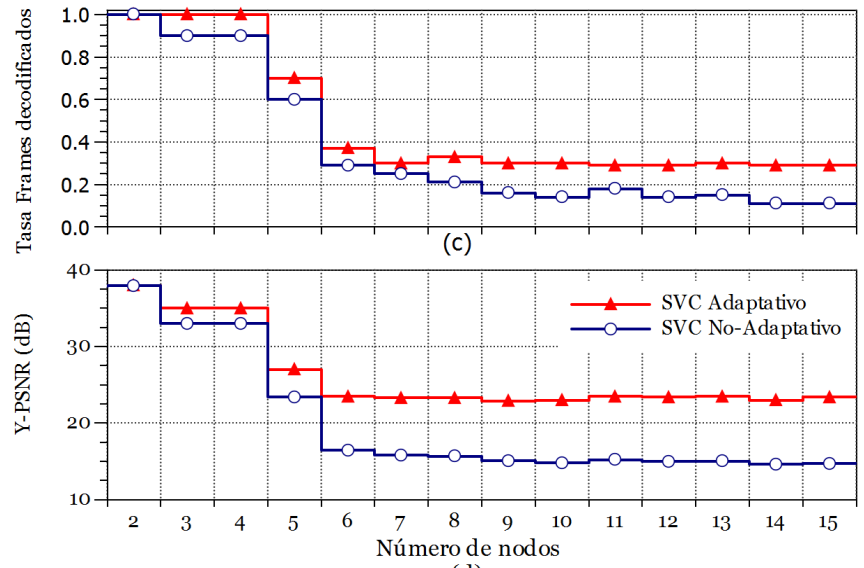

(d)

Figura 9. Resultados del escenario 1: (a) ancho de banda disponible, (b) paquetes perdidos, (c) tasa de fotogramas decodificados y (d) PSNR, en función de la longitud de la ruta.

Tal como se puede observar en la Fig. 9a, cuando la ruta entre la fuente y el receptor tiene 2 nodos (i.e. 1 salto), todas las capas SVC del video pueden ser transmitidas. Esto se debe a que el ancho disponible en la red es suficiente para soportar la tasa de bits necesaria para transmitir la capa más alta (1066 Kbps para la capa 19) y sus capas inferiores. Por lo tanto, todos los fotogramas son satisfactoriamente decodificados (tasa de fotogramas decodificados igual a 1) y la calidad del video recibido corresponde a la máxima calidad que puede ser alcanzada (38dB). Sin embargo, el ancho de banda disponible disminuye a medida que el número de nodos que componen la ruta aumenta. De tal manera que, ya no es posible transmitir todas las capas y la fuente de tráfico debería ajustar su tasa de bits. Por esta razón, cuando la ruta entre fuente y receptor tiene 3 o 4 nodos, la fuente de video máximo puede transmitir la capa $4\left(\mathrm{~T}_{4} \mathrm{Q}_{0}\right)$ y sus capas inferiores. El $100 \%$ de los fotogramas son decodificados satisfactoriamente, pero con una calidad más baja (Y-PSNR de $35 \mathrm{~dB}$ ). En cambio, cuando la fuente de video no adapta su tasa de bits (curva SVC-NoAdaptativo) algunos paquetes se pierden (aproximadamente el $10 \%)$. Sin embargo, para este porcentaje de pérdidas, la calidad del video se ve muy poco afectada debido, principalmente, a la robustez de la codificación SVC. Cuando la ruta tiene más de 5 nodos, el ancho de banda disponible disminuye drásticamente a $280 \mathrm{Kbps}$. Con este ancho de banda solo podría ser transmitida la capa $3\left(\mathrm{~T}_{3} \mathrm{Q}_{0}\right)$ y sus capas inferiores, de acuerdo a los datos de la Tabla II. En la Fig. 9b se puede observar que la estrategia de SVC adaptativa permite que la fuente ajuste su tasa de transmisión y se evite la congestión de la red. Por lo tanto, el porcentaje de paquetes perdidos no excede el $10 \%$ y el PSNR obtenido es de aproximadamente $23 \mathrm{~dB}$ (ver Fig. 9d). Por el contrario, usando la estrategia de transmisión SVC no adaptativo, el porcentaje de paquetes perdidos alcanza el $50 \%$ (casi 5 veces más que usando SVC adaptativo). Por lo tanto, la tasa de fotogramas decodificados disminuye a 0.1 y la calidad del video recibido a $14 \mathrm{~dB}$ (alrededor de $9 \mathrm{~dB}$ de diferencia con SVC adaptativo) en el peor caso.

\section{B. Escenario 2: topología móvil}

El segundo escenario es una red móvil de 30 nodos en un área de $1000 \mathrm{~m} \times 1000 \mathrm{~m}$. Los nodos se mueven hacia un destino aleatorio a una velocidad entre 0.1 y $2 \mathrm{~m} / \mathrm{s}$. Cuando llegan al destino, los nodos hacen una pausa y luego se desplazan a otro destino. En la simulación se varía la duración de dicha pausa de 0 a 80 segundos. Por lo tanto, la velocidad relativa de los nodos también varía. Un tiempo de pausa de 0 segundos es el peor escenario, ya que los nodos están continuamente moviéndose. El flujo de video se transmite entre dos nodos (nodo fuente y receptor) seleccionados aleatoriamente. Al inicio de la simulación la fuente de tráfico empieza a transmitir a la máxima velocidad posible, es decir, 1 Mbps (máxima tasa de bits para transmitir todas las capas SVC, ver Tabla II). Además del flujo de video, 3 flujos de 10 Kbps también son transmitidos por la red como tráfico de fondo. Este tráfico es de tipo CBR (Constant Bit Rate) transmitido sobre UDP.

El propósito de este escenario es evaluar los dos esquemas de transmisión de flujos de video SVC sobre redes móviles ad hoc y medir el impacto que tiene la movilidad sobre los mismos.

La Fig. 10 muestra los resultados para el escenario móvil. En relación a la perdida de paquetes (Fig. 10a), el esquema de transmisión SVC adaptativa permite evitar la congestión en la red y que el porcentaje de paquetes perdidos no sobrepase el $11 \%$, tanto para los escenarios de alta movilidad (tiempo de pausa baja) como para los de baja movilidad.

Con respecto a la calidad del video, los resultados de Fig. $10 \mathrm{~b}$ muestran que, para altos niveles de movilidad, las dos estrategias de transmisión tienen una disminución significativa en el número de fotogramas decodificados satisfactoriamente. En el caso de SVC adaptativo, esto se debe principalmente a la extracción de capas temporales del flujo SVC, llevada a cabo por la fuente de tráfico para adaptar su tasa de transmisión al ancho de banda disponible. Por el contrario, la baja tasa de fotogramas decodificados usando SVC No-adaptativo se debe tanto al alto porcentaje de paquetes perdidos, como a la eliminación de NALUs por llegar con excesivo retardo.

Finalmente, se observa una importante mejora en la calidad del video recibido usando SVC adaptativo frente a la otra alternativa (ver Fig. 10c). Para el peor caso (tiempo de pausa igual a 0 segundos), hay una mejora en el PSNR de $9 \mathrm{~dB}$, mientras que con bajos niveles de movilidad se obtiene una mejora de entre $9 \mathrm{~dB}$ y $13 \mathrm{~dB}$.

Aunque la movilidad de los nodos afecta a las dos estrategias de transmisión de video SVC, aunque no en la misma medida, claramente el esquema adaptativo permite obtener un mejor resultado en la calidad del video recibido. 
Pero además ayuda a evitar o a reducir la congestión en la red, minimizando el impacto sobre otros flujos de tráfico que se transmitan por la red.

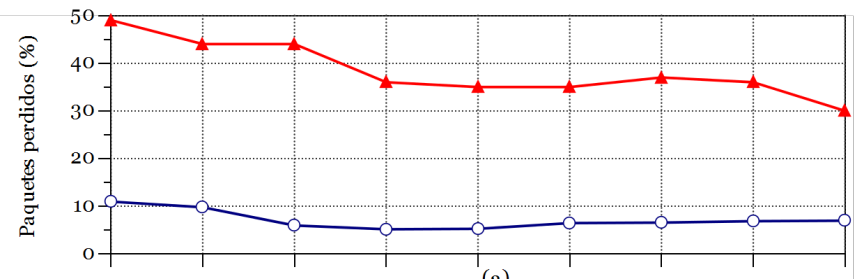

(a)
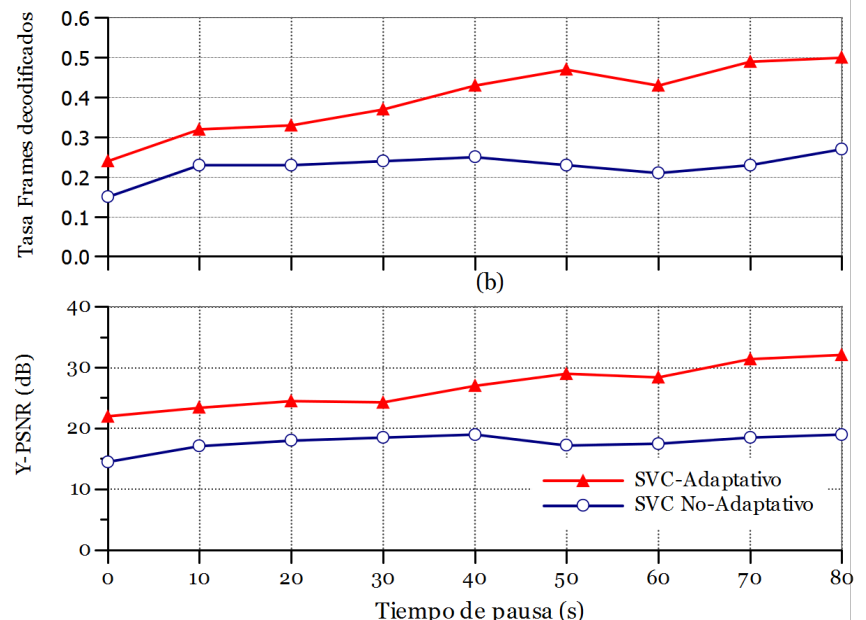

(c)

Figura 10. Resultados del escenario móvil: (a) paquetes perdidos, (b) tasa de fotogramas decodificados y (c) PSNR, como función del tiempo de pausa.

\section{CONCLUSIONES}

En este artículo se analizaron dos esquemas de transmisión de flujos de video SVC sobre redes móviles ad hoc. Por una parte se analiza la estrategia de transmisión adaptativa de videos codificados en SVC, en la cual la fuente de video es capaz de ajustar su tasa de bits de acuerdo al ancho de banda disponible. Esta alternativa fue evaluada frente a la estrategia tradicional de transmisión de flujos de video en la cual la fuente envía sus datos a una tasa constante.

Con el fin de proveer los mecanismos necesarios para que una red móvil ad hoc pueda manejar ciertos niveles de QoS, así como de un mecanismo inter-capas para que la aplicación pueda determinar la disponibilidad de recursos en la red, se desarrolló un protocolo de encaminamiento para redes móviles orientado a la trasmisión de contenido multimedia. También, se implementó una plataforma de simulación que permite desarrollar experimentos que involucren la transmisión de videos SVC sobre redes móviles ad hoc. La integración de estas dos soluciones dentro del simulador de redes NS-2, provee una plataforma eficiente que permitirá explorar nuevos esquemas de transmisión, así como la integración con otros protocolos adaptativos como DCCP, TRFC.

Los resultados de las simulaciones muestran que la estrategia adaptativa permite reducir la congestión de la red y la pérdida de paquetes, mediante la reducción de la tasa de transmisión de acuerdo a la variación del ancho de banda disponible. Esta adaptación se hace eliminando o añadiendo capas del flujo de video SVC. La evaluación de la calidad de los videos recibidos, demuestra que, aunque con el esquema adaptativo se transmiten menos datos del video, es mejor estrategia que enviar la información completa del video a una tasa constante. También se ha demostrado que la integración de nuestra plataforma de simulación y el protocolo desarrollado, construyen una herramienta útil para el estudio de la transmisión de video SVC sobre redes móviles ad hoc. La plataforma de simulación desarrollada se puede descargar del siguiente enlace [25].

Finalmente, como trabajo futuro, se planea abordar el análisis de la eficiencia energética del protocolo de encaminamiento desarrollado. Así como también, implementar mejoras en la búsqueda de rutas por medio de la estimación de varias métricas como el retardo o la energía. De igual manera, se planea complementar el análisis de la trasmisión de videos sobre redes ah doc, por medio de la evaluación de la calidad subjetiva (QoE, Quality of Experience) de los videos.

\section{REFERENCIAS}

[1] M. Conti and S. Giordano, 'Mobile ad hoc networking: milestones, challenges, and new research directions', IEEE Communications Magazine, vol. 52, no. 1, pp. 85-96, 2014.

[2] H. A. Duran-Limon, M. Siller, M. Hernandez-Ochoa, C. Quevedo, and V. Robles, 'A Network QoS Framework for Real-time Event Systems in highly Mobile Ad-hoc Environments', Journal of Applied Research and Technology, vol. 12, no. 3, pp. 343-358, 2014.

[3] ITU-T, 'Rec. H.264 \& ISO/IEC 14496-10 AVC. Advanced Video Coding for Generic Audiovisual Services'. 2013.

[4] J. Famaey, S. Latre, N. Bouten, W. Van de Meerssche, B. De Vleeschauwer, W. Van Leekwijck, and F. De Turck, 'On the merits of SVC-based HTTP Adaptive Streaming', in 2013 IFIP/IEEE International Symposium on Integrated Network Management (IM 2013), 2013, pp. 419-426.

[5] H. Kalva, V. Adzic, and B. Furht, 'Comparing MPEG AVC and SVC for adaptive HTTP streaming', in 2012 IEEE International Conference on Consumer Electronics (ICCE), 2012, pp. 158-159.

[6] S. Xiang, L. Cai, and J. Pan, 'Adaptive scalable video streaming in wireless networks', in Proceedings of the 3rd Multimedia Systems Conference, New York, NY, USA, 2012, pp. 167-172.

[7] ISO/IEC, 'Information technology - Dynamic adaptive streaming over HTTP (DASH) - Part 1: Media presentation description and segment formats', ISO/IEC 23009-1:2014, 2014.

[8] M. Sanna and E. Izquierdo, 'Live Scalable Video Streaming on Peer-toPeer Overlays with Network Coding', Latin America Transactions, IEEE (Revista IEEE America Latina), vol. 11, no. 3, pp. 962-968, 2013.

[9] M. N. Alabdulkarim and N.-E. Rikli, 'QoS Provisioning for H.264/SVC Streams over Ad-Hoc ZigBee Networks Using Cross-Layer Design', in 8th International Conference on Wireless Communications, Networking and Mobile Computing (WiCOM), 2012, pp. 1-8.

[10] E. Yaacoub, F. Filali, and A. Abu-Dayya, 'QoE Enhancement of SVC Video Streaming over Vehicular Networks Using Cooperative LTE/802.11p Communications', IEEE Journal of Selected Topics in Signal Processing, vol. PP, no. 99, pp. 1-1, 2014.

[11] P. McDonagh, C. Vallati, A. Pande, P. Mohapatra, P. Perry, and E. Mingozzi, 'Investigation of scalable video delivery using H. 264 SVC on an LTE network', in Wireless Personal Multimedia Communications (WPMC), 2011 14th International Symposium on, 2011, pp. 1-5.

[12] M. Halloush, H. R. Al-Zoubi, Z. Al-Qudah, and O. Alkofahi, 'The Performance of MANET Routing Protocols for Scalable Video Communication', Communications and Network, vol. 05, no. 02, pp. 119-125, 2013.

[13] O. Ben Rhaiem and L. Chaari Fourati, 'Routing protocols performance analysis for scalable video coding (SVC) transmission over mobile adhoc networks', in 2013 IEEE International Conference on Signal and Image Processing Applications (ICSIPA), 2013, pp. 197-202.

[14] C. Lal, V. Laxmi, and M. S. Gaur, 'QoS-aware routing for transmission of H.264/SVC encoded video traffic over MANETs', in 2013 19th Asia- 
Pacific Conference on Communications (APCC), 2013, pp. 104-109.

[15] D. Radu, J. Yi, B. Parrein, and others, 'QoE enhancement for $\mathrm{H}$. 264/SVC video transmission in MANET using MP-OLSR protocol', in Proceedings of ISIVC 2012, the 6th International Symposium on signal, Image, Video and Communications, 2012, pp. 1-4.

[16] W.-P. Lai and E.-C. Liou, 'A novel packet priority scheme and mapping algorithm for scalable video over wireless ad-hoc networks', in Wireless and Optical Communication Conference (WOCC), 2013 22nd, 2013, pp. 366-371.

[17] B. Görkemli and A. M. Tekalp, 'Adaptation strategies for MGS scalable video streaming', Signal Processing: Image Communication, vol. 27, no. 6, pp. 595-611, Jul. 2012.

[18] H. Schwarz, D. Marpe, and T. Wiegand, 'Overview of the Scalable Video Coding Extension of the H.264/AVC Standard', IEEE Transactions on Circuits and Systems for Video Technology, vol. 17, no. 9, pp. 1103-1120, 2007.

[19] A. Detti, G. Bianchi, C. Pisa, F. S. Proto, P. Loreti, W. Kellerer, S. Thakolsri, and J. Widmer, 'SVEF: an open-source experimental evaluation framework for H.264 scalable video streaming', in IEEE Symposium on Computers and Communications, 2009, pp. 36-41.

[20] T. A. Le and H. Nguyen, 'End-to-end transmission of scalable video contents: performance evaluation over EvalSVC - a new open-source evaluation platform', Multimed Tools Appl, vol. 72, no. 2, pp. 12391256, Sep. 2014.

[21] C.-H. Ke, 'myEvalSVC-an Integrated Simulation Framework for Evaluation of H. 264/SVC Transmission', KSII Transactions on Internet and Information Systems (TIIS), vol. 6, no. 1, pp. 377-392, 2012.

[22] Moving Pictures Experts Group and ITU-T Video Coding Experts Group, 'H. 264/SVC reference software (JSVM 9.19.14) and Manual'. Moving Pictures Experts Group and ITU-T Video Coding Experts Group, 2011.

[23] Wilder Castellanos, Patricia Acelas, Pau Arce, and Juan C. Guerri, 'Evaluation of a QoS-Aware Protocol with Adaptive Feedback Scheme for Mobile Ad Hoc Networks', presented at the 6th International ICST Conference on Heterogeneous Networking for Quality, Reliability, Security and Robustness, QShine 2009, Las Palmas, Gran Canaria, 2009, pp. 120-127.

[24] 'Xiph.org Video Test Media'. [Online]. Available: https://media.xiph.org/video/derf/. [Accessed: 10-Jan-2015].

[25] W. Castellanos, 'SVCEval-RA - An Evaluation Framework for Adaptive Scalable Video Streaming', SourceForge Project, 18-Aug2014. [Online]. Available: http://sourceforge.net/projects/svceval-ra/. [Accessed: 14-Jan-2015]. 